\title{
Partial Cystectomy
}

National Cancer Institute

\section{Source}

National Cancer Institute. Partial Cystectomy. NCI Thesaurus. Code C51636.

The surgical removal of a portion of a cyst. 